\title{
The NIEP
}

\author{
Charles R. Johnson, Carlos Marijuán, Pietro \\ Paparella and Miriam Pisonero
}

\begin{abstract}
The nonnegative inverse eigenvalue problem (NIEP) asks which lists of $n$ complex numbers (counting multiplicity) occur as the eigenvalues of some $n$-by- $n$ entry-wise nonnegative matrix. The NIEP has a long history and is a known hard (perhaps the hardest in matrix analysis?) and sought after problem. Thus, there are many subproblems and relevant results in a variety of directions. We survey most work on the problem and its several variants, with an emphasis on recent results, and include 130 references. The survey is divided into: a) the single eigenvalue problems; b) necessary conditions; c) low dimensional results; d) sufficient conditions; e) appending 0's to achieve realizability; f) the graph NIEP's; g) Perron similarities; and h) the relevance of Jordan structure.
\end{abstract}

Mathematics Subject Classification (2010). Primary 15A18, 15A29, 15A42.

Keywords. Nonnegative matrices, Nonnegative inverse eigenvalue problem (NIEP), Symmetric realizability.

\section{Introduction}

The Nonnegative Inverse Eigenvalue Problem (NIEP) asks which collections of $n$ complex numbers (counting multiplicities) occur as the eigenvalues of an $n$-by- $n$ matrix, all of whose entries are nonnegative real numbers. This is a long standing problem that is very difficult and, perhaps, the most prominent problem in matrix analysis. Unlike many other inverse eigenvalue problems, tools are limited, and seemingly small results are very welcome. Nonetheless, the problem is very attractive and has been attacked by many excellent researchers (see references). There have been prior surveys of work on the NIEP $([129,93,4,28,17,21,118$, , but none recently. With a great deal of important recent activity, a new survey, emphasizing this activity, will be welcome to anyone considering inquiry in this area. Our intent here is to be broad and informal. 
Another intriguing aspect of the NIEP is how many avenues can be taken to gain insight into it. Thought about it spawns endless challenging and worthy specific questions. An example is how many interesting variations there are. The real NIEP (R-NIEP) restricts the question to real spectra, and the symmetric NIEP (S-NIEP) further restricts to real spectra that are realizable by symmetric nonnegative matrices. It is known [49] that the row stochastic NIEP (restriction to nonnegative matrices with row sums 1) is equivalent to the general NIEP, but the doubly stochastic NIEP (DS-NIEP), restriction to matrices with both row and column sums 1 , is properly more restrictive, but no less difficult. Of course, there are variations on the DSNIEP, as well: the real DS-NIEP and the symmetric DS-NIEP. The Jordan NIEP (J-NIEP) asks about possible Jordan canonical forms, when there are repeated eigenvalues, and the diagonalizable NIEP (D-NIEP) is the special case in which the realizing matrix is diagonalizable. Finally, there are graph NIEP's when we consider only nonnegative matrices with a given (directed or undirected) graph $G$ (G-NIEP), or only matrices subordinate to same 0-pattern. Further particularizations may also be imagined, but these are the primary ones, so far. We should also mention that the NIEP and each variation also has a trace 0 version. This considers only spectra, the sum of whose elements is 0 , or, equivalently, nonnegative matrices, each of whose diagonal entries is 0 . In some cases, this is sufficiently restrictive, so as to make the problem easier.

We denote by $\mathrm{NIEP}_{n}$ the set of all spectra enjoyed by some $n$-by- $n$ nonnegative matrix. Such a spectrum is called realizable and a nonnegative matrix with the spectrum is called a realization or realizing matrix. The notation and terminology are similar for NIEP variants. A solution to the NIEP (or variants), for a particular $n$, is an "explicit" description of $\mathrm{NIEP}_{n}$, viewed as a subset of vectors in $\mathbb{C}^{n} . \mathrm{NIEP}_{n}$ is a closed set that is connected, and even star-shaped from the origin (or from the all 1's vector $e$ ). The set is also semi-algebraic, but is not generally convex. If $\Lambda=\left\{\lambda_{1}, \ldots, \lambda_{n}\right\}$ is a proposed spectrum, we denote by $\operatorname{Tr}(\Lambda)$ the sum of its components, counting multiplies. Other power sums $s_{k}(\Lambda)=\sum_{i=1}^{n} \lambda_{i}^{k}$, called $k$-th moments, are also important, and we usually reserve $\lambda_{1}$ as the spectral radius of $\Lambda$.

The Perron-Frobenius theory of nonnegative matrices (e.g. [44]) provides several simple, but important, necessary conditions for the NIEP (and variations). These basic necessary conditions are presented in Section 3 , followed by several more subtle necessary conditions. The "JLL" conditions are now essential to work on the problem.

The NIEP has a long history since its proposal by Kolmogorov 63], and there are many substantial results. We begin at the natural starting point of the single eigenvalue problem (Section 2) for both the row stochastic NIEP and the DS-NIEP: which individual complex numbers occur in the spectra of row stochastic and of doubly stochastic matrices. The former is solved, but there have been important advances in the description of the solution and in 
realizations. The latter is unsolved, beyond $n=4$, though there have been important recent developments.

Section 4 summarizes low-dimensional complete results for certain NIEP variants. General sufficient conditions for the realizability of spectra are given in section 5 It has long been known that $n$-fold spectra that meet simple necessary conditions, but are not realizable, may be made realizable by appending of 0 eigenvalues. Information about this phenomenon is given in section 6 . We turn to what is known about the graph-NIEP's in section 7. The new idea of Perron similarities - studying the diagonalizable NIEP's via the diagonalizing similarities - is discussed in section 8, and the role of Jordan structure in the NIEP and R-NIEP in section 9.

\section{The Single Eigenvalue Problems}

In 63, Kolmogorov posed the problem, denoted by SISEP (Stochastic Inverse Single Eigenvalue Problem), of characterizing the subset of the complex plane, denoted by $\Theta_{n}$, consisting of the individual eigenvalues of all $n$-by- $n$ stochastic matrices.

It can easily be verified that for each $n \geq 2$, the region $\Theta_{n}$ is closed, inscribed in the unit-disc, star-convex (with star-centers at zero and one), and symmetric with respect to the real-axis. Furthermore, it is clear that $\Theta_{n} \subseteq$ $\Theta_{n+1}, \forall n \in \mathbb{N}$. In view of these properties, each region $\Theta_{n}$ is determined by its boundary, which consists of so-called extremal numbers, i.e., $\partial \Theta_{n}=\{\lambda \in$ $\left.\Theta_{n}: \alpha \lambda \notin \Theta_{n}, \forall \alpha>1\right\}$.

In pursuit of characterizing $\Theta_{n}$, Dmitriev and Dynkin [25] (and independently Karpelevich [58]) showed that if $\lambda=a+b i \in \Theta_{n}$ and $b \neq 0$, then

$$
a+|b| \tan \left(\frac{\pi}{n}\right) \leq 1
$$

Let $A$ be a nonnegative matrix of order $n$ with spectral radius $\rho$ and associated directed graph $G$. Let $m$ be the length of the longest simple circuit of $G$. Kellogg and Stephens [60, Theorem 1] showed that if $m=2$, all eigenvalues of $A$ are real. If $2<m \leq n$, and if $\lambda=a+b i$ is an eigenvalue of $A$, then

$$
a+|b| \tan \left(\frac{\pi}{m}\right) \leq \rho .
$$

For further results on this topic, see [51.

Karpelevich [58, Theorem B], expanding on the work of Dmitriev and Dynkin in [26], resolved SISEP by showing that the boundary of $\Theta_{n}$ consists of curvilinear arcs (called K-arcs), whose points satisfy a polynomial equation that is determined by the endpoints of the arc (which are consecutive roots of unity). The statement of this theorem is unwieldy, but Ito 47, Theorem 2] provides a useful simplification of the result.

Noticeably absent in the Karpelevich Theorem (and other works) are realizing-matrices (i.e., a matrix whose spectrum contains a given eigenvalue) for points on the K-arcs. Dmitriev and Dynkin [26, Basic Theorem] give a schematic description of such matrices for points on the boundary of 
$\Theta_{n} \backslash \Theta_{n-1}$ and Swift [129, $\left.\S 2.2 .2\right]$ provides such matrices for $3 \leq n \leq 5$. Recently, Johnson and Paparella [56] provide, for every $n$ and for each K-arc, a single parametric stochastic matrix that realizes the entire K-arc as the parameter runs from 0 to 1 .

For $n \in \mathbb{N}$, denote by $\Pi_{n}$ the convex-hull of the $n^{\text {th }}$ roots-of-unity, i.e.,

$$
\Pi_{n}=\left\{\sum_{k=0}^{n-1} \alpha_{k} \exp (2 \pi i k / n) \in \mathbb{C}: \alpha_{k} \geq 0, \sum_{k=0}^{n-1} \alpha_{k}=1\right\} .
$$

Denote by $\Omega_{n}$ the subset of the complex-plane containing all single eigenvalues of all $n$-by- $n$ doubly stochastic matrices. Perfect and Mirsky [103] conjectured that $\Omega_{n}=\bigcup_{k=1}^{n} \Pi_{k}$ and proved their conjecture when $1 \leq n \leq 3$. Levick et al. [76] proved the Perfect-Mirsky conjecture when $n=4$ but a counterexample when $n=5$ was given by Mashreghi and Rivard [88. Recently, Levick et al. conjectured that $\Omega_{n}=\Theta_{n-1} \cup \Pi_{n}$ ([76. Conjecture 1]), but there is computational evidence that suggests that the $n=5$ case is either a rare exception, or the only exception, to Perfect-Mirsky.

\section{Necessary Conditions for the NIEP's}

For a proposed spectrum $\Lambda=\left\{\lambda_{1}, \lambda_{2}, \ldots, \lambda_{n}\right\}$, repeats allowed, to be (NIEPrealizable, a number of necessary conditions are known. The most basic of these follow from the fact that a nonnegative matrix has real entries and nonnegative trace, and from the Perron-Frobenius theory of nonnegative matrices. (We assume the reader is familiar with the Perron-Frobenius theory, which is recounted, for example in [44.) For simplicity, we label these according to simple titles. Since a nonnegative matrix is real, it must have the eigenvalues of a real matrix, i.e. the characteristic polynomial must have real coefficients, or, as a list

(Reality)

$$
\bar{\Lambda}=\Lambda \text {. }
$$

Since the trace of a matrix is the sum of the eigenvalues and the trace of a nonnegative matrix is nonnegative, we also have

$$
\operatorname{Tr} \Lambda=\sum_{i=1}^{n} \lambda_{i} \geq 0
$$

Of course, it follows that if $\Lambda$ is realizable and $\operatorname{Tr}(\Lambda)=0$, then all diagonal entries of any realizing matrix must be 0 . Higher power sums are also nonnegative as they are traces of positive integral powers of nonnegative matrices:

(k-th moment)

$$
s_{k}(\Lambda)=\lambda_{1}^{k}+\cdots+\lambda_{n}^{k} \geq 0, \quad k \geq 1 .
$$

According to the Perron-Frobenius theory, the spectral radius of a nonnegative matrix must, itself, be an eigenvalue, so among the eigenvalues $\Lambda$ 
must be a nonnegative one, at least as big in absolute value as any others. Without loss of generality, this one may be taken to be $\lambda_{1}$. So, we have

$$
\lambda_{1} \geq\left|\lambda_{i}\right|, \quad i=2, \ldots, n,
$$

and if any of the other eigenvalues are the same as $\lambda_{1}$, any realizing matrix must be reducible. Moreover, if all the inequalities are strict, any irreducible realizing matrix must be primitive.

The above conditions are necessary for all the NIEP's, but there are some necessary conditions for particular NIEP's.

Further necessary conditions are more subtle, but many have been noticed. The most ubiquitous of these was noticed independently in [49] and in [78] and is usually referred to as the JLL conditions. They generalize the trace condition and follow from the fact that every power of a nonnegative matrix is nonnegative and that positive diagonal entries must contribute to positive diagonal entries in powers $\left(e . g . s_{p}(\Lambda)>0\right.$ implies $s_{p q}(\Lambda)>0$ for integers $p \geq 1$ and $q \geq 1$ ). The general (quantitative) version is

$$
\left(s_{k}(\Lambda)\right)^{m} \leq n^{m-1} s_{k m}(\Lambda), \quad k, m=1,2, \ldots .
$$

Let $A$ be an $n$-by- $n$ real matrix with spectrum $\Lambda=\left\{\lambda_{1}, \ldots, \lambda_{n}\right\}$. Denote the principal submatrix of $A$ lying in the rows and columns given by the index set $\alpha \subseteq\{1, \ldots, n\}$ by $A[\alpha]$. Define the $k$-th elementary symmetric function

$$
E_{k}(\Lambda)=\sum_{1 \leq i_{1}<\cdots<i_{k} \leq n} \lambda_{i_{1}} \cdots \lambda_{i_{k}}
$$

and the $k$-th Newton coefficient

$$
c_{k}(\Lambda)=\frac{E_{k}(\Lambda)}{\left(\begin{array}{l}
n \\
k
\end{array}\right)}, \quad k=1, \cdots, n, \text { with } c_{0} \equiv 1 .
$$

Since $E_{k}(\Lambda)=\sum_{|\alpha|=k} \operatorname{det} A[\alpha]$, as well, $c_{k}(\Lambda)$ may be viewed as the average value of the $k$-by- $k$ principal minors of $A$. The spectrum $\Lambda$ is called Newton if

$$
c_{k}(\Lambda)^{2} \geq c_{k-1}(\Lambda) c_{k+1}(\Lambda), \quad k=1, \ldots, n-1,
$$

and these inequalities are referred to as the Newton inequalities [43, 52].

They hold for $\Lambda \geq 0$ 97], $\Lambda \subset \mathbb{R}$ [1], and they are valid for real diagonal matrices, diagonalizable matrices with real spectra (the $c_{k}$ are invariant under similarity), and matrices with real spectra. In [43 it was proved that the Newton inequalities also hold for $M$-matrices and, thus, inverse $M$-matrices and it was observed that if $A$ is a nonnegative matrix with spectral radius $\rho(A)$, then $\rho(A) I-A$ is an M-matrix and, therefore, must satisfy the Newton 
inequalities. If we denote its spectrum $\left\{\rho(A)-\lambda_{1}, \ldots, \rho(A)-\lambda_{n}\right\}$ by $\rho(A)-\Lambda$, then we have new necessary conditions:

$$
c_{k}(\rho(A)-\Lambda)^{2} \geq c_{k-1}(\rho(A)-\Lambda) c_{k+1}(\rho(A)-\Lambda), \quad k=1, \ldots, n-1 .
$$

In 130 the authors did not focus the attention directly on the spectrum but on the coefficients of the characteristic polynomial. Thus, the NIEP that they consider is: given real numbers $k_{1}, k_{2}, \ldots, k_{n}$, find necessary and sufficient conditions for the existence of a nonnegative matrix of order $n$ with characteristic polynomial $x^{n}+k_{1} x^{n-1}+k_{2} x^{n-2}+\cdots+k_{n}$.

The coefficients of the characteristic polynomial are closely related to the cyclic structure of the weighted digraph associated with the matrix $A$, as established by the Coefficients Theorem [23, Theorem 1.3*]. The authors 130] introduce graphic tools to study the NIEP from the characteristic polynomial and use the following method: if $P(x)$ is a realizable polynomial, in the sense that there exists a nonnegative matrix with characteristic polynomial $P(x)$, we try to maximize each coefficient $k_{j}$ as a function of the previous coefficients, preserving the realizability for a polynomial of degree $n$ with the same previous coefficients. Note that $k_{j}$ is a continuous function (sum of determinants) of the entries of a nonnegative matrix $A$ realizing the polynomial $P(x)$ and that, as the previous coefficients are bounded above, then the entries of $A$ involved in the expression of $k_{j}$ are also bounded above (Coefficients Theorem); therefore, this maximum is attained. In this way, new necessary conditions on the three first coefficients are obtained 130, Theorem $3]$ :

$$
k_{1} \leq 0
$$

(TAAMP)

$$
k_{2} \leq \frac{n-1}{2 n} k_{1}^{2}
$$

$k_{3} \leq \begin{cases}\frac{n-2}{n}\left(k_{1} k_{2}+\frac{n-1}{3 n}\left(\left(k_{1}^{2}-\frac{2 n k_{2}}{n-1}\right)^{\frac{3}{2}}-k_{1}^{3}\right)\right) & \text { if } \quad \frac{(n-1)(n-4)}{2(n-2)^{2}} k_{1}^{2}<k_{2}, \\ k_{1} k_{2}-\frac{(n-1)(n-3)}{3(n-2)^{2}} k_{1}^{3} & \text { if } \quad k_{2} \leq \frac{(n-1)(n-4)}{2(n-2)^{2}} k_{1}^{2} .\end{cases}$

Another necessary condition in terms of the $k$-th moments $s_{k}=\operatorname{Tr}\left(A^{k}\right)$, $k=1,2,3$, is obtained in [22, Theorem 3]:

$$
\Phi:=n^{2} s_{3}-3 n s_{1} s_{2}+2 s_{1}^{3}+\frac{n-2}{\sqrt{n-1}}\left(n s_{2}-s_{1}^{2}\right)^{3 / 2} \geq 0 .
$$

The necessary conditions previous to (JLL) are not independent. In fact, ( $k$-th moment) implies (Perron) [34] and also (Reality) [78]. For $k=1$, (JLL) is reduced to $\left(s_{1}(\Lambda)\right)^{m} \leq n^{m-1} s_{m}(\Lambda)$, and so, if $s_{1}(\Lambda) \geq 0$, then (JLL) implies ( $k$-th moment). 
On the other hand, if we denote the two bounds given for the coefficient $k_{3}$ in (TAAMP) by $k_{3}^{\max 1}$ and $k_{3}^{\max 2}$, we can use the Newton identities

$$
s_{m}+k_{1} s_{m-1}+\cdots+k_{m-1} s_{1}+k_{m} m=0, \quad k, m=1,2,3,
$$

to rewrite the condition (CL) in the form

$$
\Phi=3 n^{2}\left(k_{3}^{\max 1}-k_{3}\right) .
$$

In the first case, i.e., if $k_{2}>\frac{(n-1)(n-4)}{2(n-2)^{2}} k_{1}^{2}$, we have that $\Phi \geq 0$ implies $k_{3}^{\max 1} \geq k_{3}$. In the contrary case, $\Phi=3 n^{2}\left(k_{3}^{\max 1}-k_{3}\right) \geq 3 n^{2}\left(k_{3}^{\max 2}-k_{3}\right) \geq 0$. So, in any case, the condition on the coefficient $k_{3}$ is stronger than the condition (CL).

In 43 it was proved that ( $k$-th moment), (JLL) and $(\mathrm{H})$ are mutually independent. In [84, Theorem 11] that (JLL) for $k=1$ and $m=2, s_{1}(\Lambda)^{2} \leq$ $n s_{2}(\Lambda)$, the first $(\mathrm{H}), c_{1}(\rho-\Lambda)^{2} \geq c_{2}(\rho-\Lambda)$, and (TAAMP) over the second coefficient, $k_{2}(\Lambda) \leq \frac{n-1}{2 n} k_{1}(\Lambda)^{2}$, are equivalent. But, in general, at least (JLL) and (TAAMP) are independent of the others, and this tandem implies ( $k$-th moment). Some examples: the spectra $\{20,-18,5 \sqrt{2} \pm 5 \sqrt{2} i\}$ and $\{1,1,1,0,0\}$ satisfy (JLL), (H) and (CL), but not (TAAMP); the spectra $\{2,-2,-2,1 \pm i\}$ and $\{3,1,1,1,1,1,-2,-2,-2,-2\}$ satisfy (H) and (TAAMP), but not (JLL). That the necessary conditions (JLL), (H) and (TAAMP) are not sufficient for the NIEP is proved by the non-realizable list $\{3,3,-\sqrt{3} \pm i\}$.

Conjecture. (JLL) and (TAAMP) imply all known necessary conditions. (It is enough to prove that (JLL) and (TAAMP) imply (H)).

In [69 a necessary condition for trace 0 and $n$ odd was obtained, given by

$$
\left(s_{2}(\Lambda)\right)^{2} \leq(n-1) s_{4}(\Lambda) .
$$

This condition has been generalized in terms of the coefficients by the following result [130, Lemma 37]: if $x^{n}+k_{p} x^{n-p}+\cdots+k_{2 p} x^{n-2 p}+\cdots+$ $k_{n}, k_{p} \neq 0$, is the characteristic polynomial of a nonnegative matrix, then

$$
k_{2 p} \leq \frac{1}{2}\left(1-\frac{1}{\lfloor n / p\rfloor}\right) k_{p}^{2} .
$$

We can use the Newton identities (1) to express the inequality (2) in terms of the $k$-th moments: if $s_{1}=\cdots=s_{p-1}=0$, we have the more general condition

$$
s_{p}^{2} \leq p\left\lfloor\frac{n}{p}\right\rfloor s_{2 p}, \quad p=1, \ldots, \frac{n}{2}
$$

that coincides with (LM) in the particular case $p=2$ and $n$ odd.

Note also that the (JLL) condition for $m=2$ is $s_{p}^{2} \leq n s_{2 p}$, and that $p\left\lfloor\frac{n}{p}\right\rfloor \leq n$. Then the expression $[2]$ is a restricted refinement of the (JLL) conditions. 


\section{Low Dimensional Results}

The NIEP for $n \leq 3$ was solved independently by Oliveira [98, Theorem (6.2)] and Loewy-London [78]. For the non-real case:

$$
\{\lambda, z, \bar{z}\} \text { is NIEP-realizable } \Longleftrightarrow z \in \lambda \Pi_{3}=\left\{\lambda z^{\prime}: z^{\prime} \in \Pi_{3}\right\}
$$

Meehan [90] solved the NIEP for $n=4$ in terms of the $k$-th moments, and Torre-Mayo et al. [130] in terms of the coefficients of the characteristic polynomial using the necessary conditions (TAAMP). For $n \geq 5$ it remains unsolved.

The R-NIEP for $n \leq 4$ was solved independently by Perfect [100], Oliveira [98, §9] and Loewy-London [78; in these cases the necessary conditions (Trace) and (Perron) are also sufficient.

Perfect [102, Theorem 4] solved the R-NIEP for $n=3$ with fixed diagonal entries: $\left\{d_{1}, d_{2}, d_{3}\right\}$ is the diagonal of a 3 -by-3 nonnegative matrix with spectra $\left\{\lambda_{1}, \lambda_{2}, \lambda_{3}\right\}$, where $\lambda_{1} \geq \lambda_{2} \geq \lambda_{3}$, if and only if

$$
0 \leq d_{i} \leq \lambda_{1} ; \quad \sum_{i=1}^{3} d_{i}=\sum_{i=1}^{3} \lambda_{i} ; \quad \sum_{i \neq j} d_{i} d_{j} \geq \sum_{i \neq j} \lambda_{i} \lambda_{j} ; \quad \max \left\{d_{i}\right\} \geq \lambda_{2} .
$$

The S-NIEP and the R-NIEP are equivalent for $n \leq 4$, and remain unsolved for $n \geq 5$. Fiedler [33, Theorem 4.8] solved the S-NIEP in the case $n=3$ with fixed diagonal entries: $\left\{d_{1}, d_{2}, d_{3}\right\}$, with $d_{1} \geq d_{2} \geq d_{3} \geq 0$, is the diagonal of a 3-by-3 symmetric nonnegative matrix with spectra $\left\{\lambda_{1}, \lambda_{2}, \lambda_{3}\right\}$, where $\lambda_{1} \geq \lambda_{2} \geq \lambda_{3}$, if and only if $\left\{\lambda_{1}, \lambda_{2}, \lambda_{3}\right\}$ majorizes $\left\{d_{1}, d_{2}, d_{3}\right\}$ and $d_{1} \geq \lambda_{2}$.

Johnson-Laffey-Loewy [50] showed that the R-NIEP and the S-NIEP are different, and Egleston-Lenker-Narayan [28] proved that they are different for $n \geq 5$.

The S-NIEP for $n=5$ has been widely studied [79, 89, 28, but not fully resolved. It is common to study it considering the number of positive eigenvalues. When there are 1, 4 or 5 positive eigenvalues the answer for the S-NIEP is straightforward. Recently, Johnson-Marijuán-Pisonero [54] resolved all cases with 2 positive eigenvalues, and they give a method, based upon the eigenvalue interlacing inequalities for symmetric matrices, to rule out many unresolved spectra with 3 positive eigenvalues. In particular, this method shows that the nonnegative realizable spectrum $\{6,3,3,-5,-5\}$ is not symmetrically realizable.

Also recently, Loewy-Spector [80, Theorem 4] characterize the case $n=$ 5 in a particular case: $\Lambda=\left\{\lambda_{1}, \lambda_{2}, \ldots, \lambda_{5}\right\}$, where $\lambda_{1} \geq \lambda_{2} \geq \cdots \geq \lambda_{5}$ and $2 s_{1}(\Lambda) \geq \lambda_{1}$, is (S-NIEP) realizable if and only if (Perron), $\lambda_{2}+\lambda_{5} \leq$ $\operatorname{Tr}(\Lambda), \lambda_{3} \leq \operatorname{Tr}(\Lambda)$. This last condition is implied by the constraint $2 s_{1}(\Lambda) \geq$ $\lambda_{1}$.

The trace 0 NIEP has also been extensively studied. Reams [107 solved the case $n=4:\left\{\lambda_{1}, \lambda_{2}, \lambda_{3}, \lambda_{4}\right\}$ is trace 0 (NIEP)-realizable if and only if $s_{1}=0, s_{2}, s_{3} \geq 0$ and $s_{2}^{2} \leq 4 s_{4}$. The case $n=5$ was first studied by Reams 107. and he gave a sufficient condition. The case $n=5$ was finally solved 
by Laffey-Meehan [70]: $\left\{\lambda_{1}, \ldots, \lambda_{5}\right\}$ is trace 0 (NIEP)-realizable if and only if $s_{1}=0, s_{2}, s_{3} \geq 0, s_{2}^{2} \leq 4 s_{4}$ and $12 s_{5}+5 s_{3} \sqrt{4 s_{4}-s_{2}^{2}} \geq 5 s_{2} s_{3}$.

Torre-Mayo et al. [130, generalize these solutions in terms of the coefficients of the characteristic polynomial: the polynomial $x^{n}+k_{p} x^{n-p}+\cdots+$ $k_{n-1} x+k_{n}$, with $2 \leq p \leq n \leq 2 p+1$, is (NIEP)-realizable if and only if

$k_{p}, \ldots, k_{2 p-1} \leq 0 ; k_{2 p} \leq \frac{k_{p}^{2}}{4} ; k_{2 p+1} \leq \begin{cases}k_{p} k_{p+1} & \text { if } k_{2 p} \leq 0, \\ k_{p+1}\left(\frac{k_{p}}{2}-\sqrt{\frac{k_{p}^{2}}{4}-k_{2 p}}\right) & \text { if } k_{2 p}>0 .\end{cases}$

Spector [127 characterized trace 0 S-NIEP realizability for $n=5$ by the conditions $\lambda_{2}+\lambda_{5} \leq 0$ and $s_{3} \geq 0$.

\section{Sufficient Conditions}

The first known sufficient condition for the NIEP, that in fact is for the RNIEP, was announced by Sulě̌manova [128] in 1949 and proved by Perfect 101] in 1953:

$$
\left.\begin{array}{r}
\Lambda=\left\{\lambda_{1}, \ldots, \lambda_{n}\right\} \text { real, } \lambda_{1} \geq|\lambda| \text { for } \lambda \in \Lambda \\
\text { and } \lambda_{1}+\sum_{\lambda_{i}<0} \lambda_{i} \geq 0
\end{array}\right\} \Longrightarrow \Lambda \text { is realizable. }
$$

Several other proofs have been given, e.g. [99]. There are several sufficient conditions for the R-NIEP that are checkable in a straightforward way, that is, one only needs to check a few algebraic inequalities, perhaps after ordering the spectrum. The authors of them are: Ciarlet in 1968 [20], Kellogg in 1971 [59, Salzmann in 1972 [112] and Fiedler in 1974 [33. Borobia in 1995 6] extended Kellogg's condition by grouping negative eigenvalues.

Other sufficient conditions for the R-NIEP involve partitions of the spectra considered, such as an immediate piece-wise extension of the Sulermanova condition. We will name this condition Suleǐmanova-Perfect [128, 101]:

$$
\left.\begin{array}{r}
\Lambda=\left\{\lambda_{1}, \lambda_{11}, \ldots, \lambda_{1 t_{1}}, \ldots, \lambda_{r}, \lambda_{r 1}, \ldots, \lambda_{r t_{r}}\right\} \text { real } \\
\lambda_{1} \geq|\lambda| \text { for } \lambda \in \Lambda \\
\text { and } \lambda_{j}+\sum_{\lambda_{j i}<0} \lambda_{j i} \geq 0 \text { for } j=1, \ldots, r
\end{array}\right\} \Longrightarrow \Lambda \text { is realizable. }
$$

Other sufficient conditions of this type are more elaborate and we will name them by their authors: Perfect 1 in 1953 [101], Soto 2 in 2003 [115] or its extension Soto $p$ in 2013 [119].

Some of the sufficient conditions besides partitions involve the knowledge of the diagonal entries of a realization of part of the spectrum. The first 
condition of this type is due to Perfect in 1955 [102]:

$$
\left.\begin{array}{r}
\Lambda=\left\{\lambda_{1}, \ldots, \lambda_{r}\right\} \cup\left\{\lambda_{11}, \ldots, \lambda_{1 t_{1}}\right\} \cup \cdots \cup\left\{\lambda_{r 1}, \ldots, \lambda_{r t_{r}}\right\} \\
\left\{\lambda_{1}, \ldots, \lambda_{r}\right\} \text { the spectrum of a nonnegative } \\
\text { matrix with diagonal } d_{1}, \ldots, d_{r}, \\
\lambda_{j i} \leq 0 \text { for } j=1, \ldots, r \text { and } i=1, \ldots, t_{j}, \\
\lambda_{1} \geq|\lambda| \text { for } \lambda \in \Lambda, \sum_{\lambda \in \Lambda} \lambda \geq 0 \\
\text { and } d_{j}+\sum_{1 \leq i \leq t_{j}} \lambda_{j i} \geq 0, j=1, \ldots, r
\end{array}\right\} \Rightarrow \Lambda \text { is realizable. }
$$

When $\lambda_{j} \geq 0$, for $j=1, \ldots, r$, we call this condition Perfect $\mathbf{2}^{+}$(see [86]). There are two equivalent conditions that extend this condition: Soto-Rojo in 2006 [122] and Soto-Rojo-Manzaneda in 2011 [123.

Other well-known sufficient conditions manipulate certain spectra to get a new realizable spectrum: Guo in 1997 [40, Theorems 2.1 and 3.1] or $C$-realizability [11] that we name the game condition [85].

In order to construct a map of sufficient conditions for the R-NIEP, Marijuán-Pisonero-Soto compared these conditions and established inclusion relations or independence relations between them, [86, 85]:

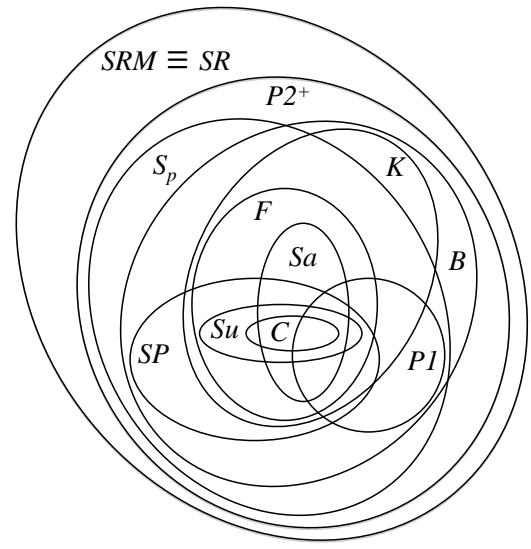

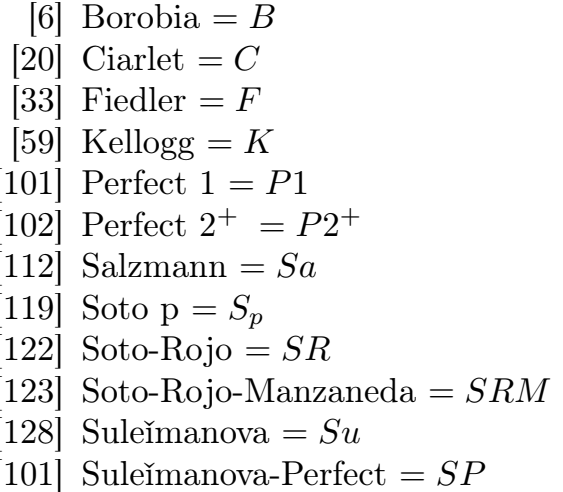

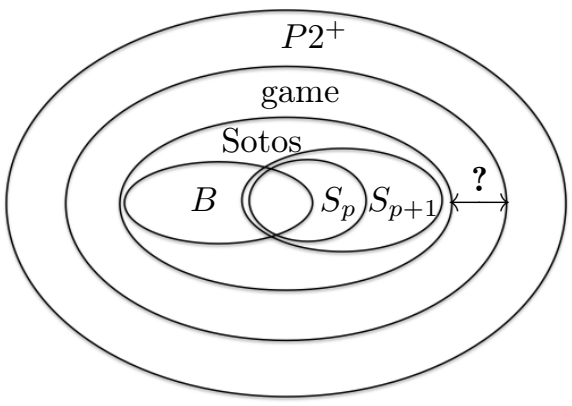

[6] Borobia $=B$

102 Perfect $2^{+}=P 2^{+}$

[119] Soto $\mathrm{p}=S_{p}$

[119] Soto $\mathrm{p}+1=S_{p+1}$

[119] Sotos $=\bigcup_{p \geq 2}$ Soto $p$

The first known sufficient condition for the S-NIEP is due to PerfectMirsky in 1965 [103] for doubly stochastic matrices, and Fiedler in 1974 
33 gave the first one for symmetric nonnegative matrices. Several sufficient conditions which were first obtained for the R-NIEP have later been shown to be valid also for the S-NIEP as well. Fiedler [33, Radwan [106] and Soto [117] showed, respectively, that Kellogg [59, Borobia [6] and Soto 2 [115] are also symmetric sufficient conditions.

Soules in 1983 [126] gave two constructive sufficient conditions for symmetric realization. The inequalities that appear in these conditions are obtained by requiring the diagonal entries of the matrix $R \operatorname{diag}\left(\lambda_{1}, \ldots, \lambda_{n}\right) R^{T}$ to be nonnegative, in which $R$ is an orthogonal matrix with a certain pattern. For a particular $R$, this condition is the Perfect-Mirsky condition.

Soto-Rojo-Moro-Borobia gave in 2007 [124] a symmetric version of the Soto-Rojo condition. Soto $p$ [119] and Soto-Rojo-Manzaneda [123] have also symmetric versions. Laffey-Šmigoc in 2007 [72] gave the symmetric realizability of a spectrum by manipulating two spectra.

Again in order to construct a map of sufficient conditions for the S-NIEP, Marijuán-Pisonero-Soto [87] compared these conditions and established inclusion relations or independence relations between them:

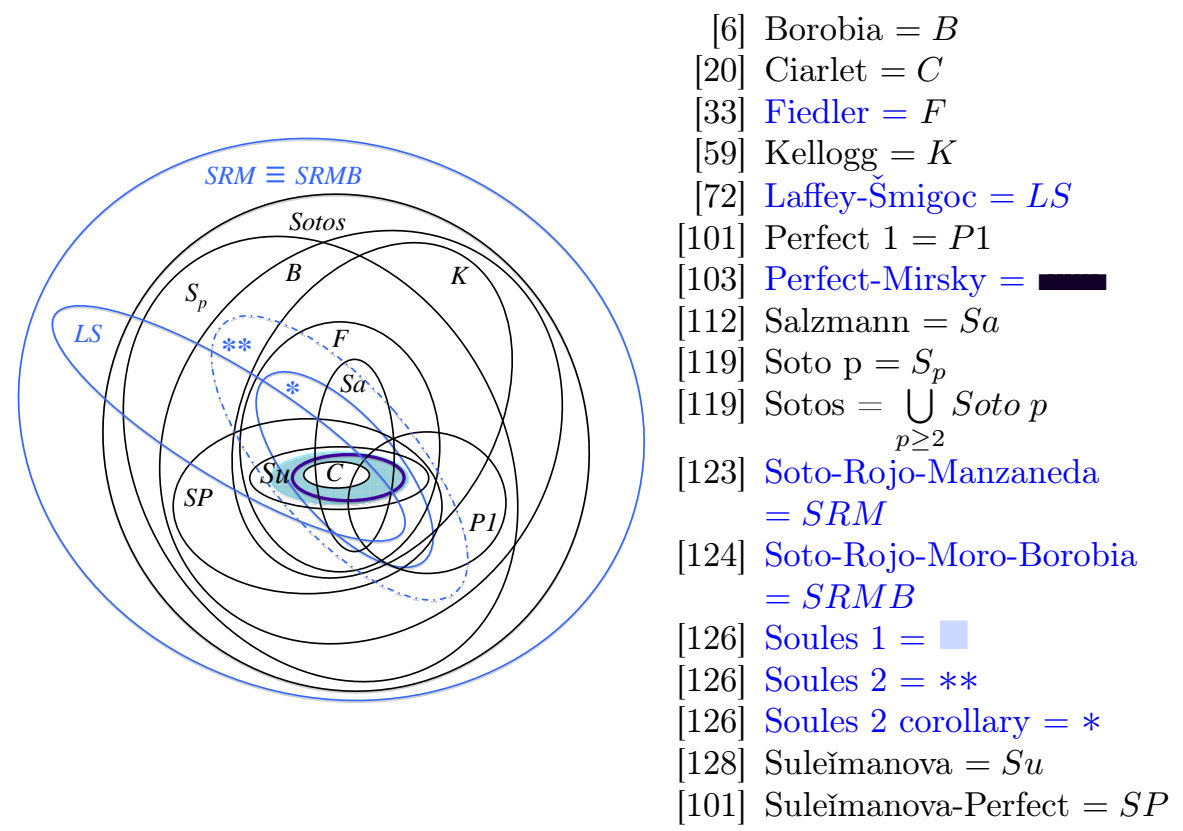

The discontinuous line for Soules 2 in the map means that we only conjecture this position for this sufficient condition.

Recently, Ellard-Šmigoc [30] have modified the Laffey-Šmigoc condition and the Soules 2 condition and they have proved the following equivalence:

Soules 2 modified $\Longleftrightarrow$ Laffey-ŠSmigoc modified $\Longleftrightarrow$ game $\Longleftrightarrow$ Sotos. 
This implies the symmetric realizably of the game condition and resolves the question on the RNIEP diagram about whether there exists something between Sotos and game.

There are also some sufficient conditions for the NIEP. Guo in 1997 40, Theorems 2.1 and 3.1] gave results about changing a realizable spectrum to obtain a realizable spectrum. Šmigoc in 2004 [113, Theorems 10 and 14] gave other such results. Finally, let us mention some generalizations of the Suleřmanova condition: Laffey-Šmigoc in 2006 [71, Theorems 1 and 3] and Borobia-Moro-Soto in 2008 [11, Theorem 3.3].

\section{Embedding Spectra, by Adding 0's, to Achieve Realizability}

Consider now spectra that meet the most basic necessary conditions for NIEP-realizability: (Perron), (Trace) and (Reality). Even when these conditions are strictly met, the proposed spectrum, $\sigma$, need not be realizable (for example $\{3,3,-\sqrt{3} \pm i\}$ ). This raises the natural question of whether the spectrum may be embedded in a larger one, that is realizable, by appending some additional eigenvalues, e.g. $\sigma \hookrightarrow \sigma \cup \tau$. If we are too liberal about what eigenvalues may be appended, this question becomes trivial. For example, realizability may always be achieved by appending a single, sufficiently large, positive eigenvalue; the Perron and trace conditions may be arbitrarily improved. Thus, some condition must be placed upon the appended eigenvalues. A natural one is that only 0 eigenvalues may be appended; now, the Perron and trace conditions are not enhanced - but, the dimension is increased. (Intermediate restrictions seem not yet to have been considered.) The increase in dimension does improve the possibility of meeting the JLL conditions.

The notion of appending 0's to "repair" a nonrealizable spectrum may and has often been viewed another way: what collections of complex numbers occur as the "nonzero part" of the spectrum of an entry-wise nonnegative matrix. It turns out that this question is quite different from and more tractable than the classical NIEP.

The first to show that appending 0's can help was [49], in which it was shown that the spectrum

$$
1, \sqrt{\frac{3}{8}} i,-\sqrt{\frac{3}{8}} i
$$

is not realizable in dimension 3 (because the single eigenvalue conditions [58] are not met, or the JLL conditions are not met), but the spectrum

$$
1, \sqrt{\frac{3}{8}} i,-\sqrt{\frac{3}{8}} i, 0
$$

is realizable in dimension 4 by the matrix

$$
\left(\begin{array}{cccc}
1 / 4 & 0 & 3 / 4 & 0 \\
1 / 4 & 1 / 4 & 0 & 1 / 2 \\
0 & 3 / 4 & 1 / 4 & 0 \\
7 / 24 & 0 & 11 / 24 & 1 / 4
\end{array}\right)
$$


Later in [12], it was shown, remarkably, that if a spectrum (with no 0's) meets the basic necessary conditions strictly, then it is the nonzero part of the spectrum of a nonnegative matrix. Sufficiently many 0's may be appended to achieve realizability. Of course, the number of 0's that need be added may be very large (not uniformly bounded in terms of $n$ ) because of JLL. Interestingly, the newish method of symbolic dynamics was used in an import way, though many easily proven matricial lemmas were needed as well. Specifically the result is

Theorem 1. The list of nonzero complex numbers $\Lambda=\left\{\lambda_{1}, \lambda_{2}, \ldots, \lambda_{n}\right\}$ is the nonzero spectrum of a primitive matrix if and only if

1. strict Perron condition: $\Lambda$ contains a positive eigenvalue of multiplicity one that is greater in absolute value than all other $\lambda_{i}^{\prime} s$;

2. reality condition: $p(t)=\prod_{i=1}^{n}\left(t-\lambda_{i}\right)$ has real coefficients; and

3. extended trace condition: $\sum_{i=1}^{n} \lambda_{i}^{m k} \geq 0$ for all $k$ and $\sum_{i=1}^{n} \lambda_{i}^{k}>0$ implies $\sum_{i=1}^{n} \lambda_{i}^{m k}>0$ for all $m$.

The necessity of these conditions is easily verified (the last one via JLL, for example), and the interesting point is that necessary conditions become sufficient when primitivity is the goal and the dimension may be arbitrarily increased. When the Perron condition is not strict it may not be possible to "save" a spectrum meeting obvious necessary conditions. The familiar example $3,3,-2,-2,-2$ is not only not realizable, but is never the nonzero part of the spectrum of a nonnegative matrix. However, both $3+\epsilon, 3,-2,-2,-2$ and $3+\epsilon, 3-\epsilon,-2,-2,-2$ are both the nonzero parts of the spectra of primitive matrices for arbitrarily small $\epsilon>0$ (though they are not realizable for $\epsilon$ small).

More recently there have been further developments about realizability after appending 0's to a spectrum. In [66] there is matricial proof of key results from [12, which is much more explicit. Though the number of 0's needed to make a spectrum realizable may be very large (and not easy to estimate from [12]), estimates have recently been given under some circumstances by bringing $s_{2}(\Lambda)$, as well as the trace, into play [71].

\section{The Graph NIEP}

Not surprisingly, realizable spectra that are, in some way extremal, are often realizable by nonnegative matrices with many 0 entries, as are many nonextremal spectra. This raises the question that, if we fix the 0-pattern of a nonnegative matrix, how is the NIEP restricted, i.e. which realizable spectra occur? A natural way to describe a particular 0-pattern is via a graph, which could be directed or undirected. For a particular graph $G$ on $n$ vertices, consider the set of nonnegative $n$-by- $n$ matrices $N(G)$ for which $A=\left(a_{i j}\right)$ 
satisfies $a_{i j}>0$ if and only if $(i, j)$ is a directed edge, $i \neq j$, of $G$. (Here, we consider an undirected edge $\{i, j\}$, if $G$ is undirected, to consist of two directed edges $(i, j)$ and $(j, i)$.) For simplicity we consider graphs without loops, and no restriction is placed by the graph upon the diagonal entries, other than nonnegativity. The G-NIEP then just asks which spectra occur among matrices in $N(G)$ ? (if we wish to emphasize the dimension, which we generally take to be implicit, we may write $N_{n}(G)$.) Of course the NIEP is just the union of the solutions to the G-NIEP's, over all directed graphs $G$. The same is true for various variations upon the NIEP. For example, the solution to the S-NIEP is just the solution to the G-NIEP, restricted to symmetric matrices, over all undirected graphs. Thus far, the G-NIEP has been considered only for undirected graphs $G$, symmetric matrices and real eigenvalues. This seems a fertile area for future work.

A prototype of the G-NIEP, though not presented in graph terms, appeared in [35], in which tridiagonal matrices were considered. Notice that the spectrum of a nonnegative tridiagonal matrix is not only necessarily real but also the spectrum of a symmetric tridiagonal nonnegative matrix. Also, tridiagonal matrices are the case in which $G$ is a path (and an edge only requires nonnegativity of the entry); off-diagonal 0 entries are important. We say that a matrix $A$ is subordinate to a graph $G$ if $G(A)$ has the same vertices as $G$ and the edges of $G(A)$ are contained among those of $G$; equivalently, for $A=\left(a_{i j}\right), a_{i j} \neq 0$ implies $\{i, j\}$ is an edge of $G$. The main result of 35] is for the path $P$ on $n$ vertices. Then, for $\lambda_{1} \geq \lambda_{2} \geq \cdots \lambda_{n}$, there is an $n$-by- $n$ nonnegative matrix $A^{T}=A$ subordinate to $P$ and with eigenvalues $\lambda_{1}, \lambda_{2}, \ldots, \lambda_{n}$ if and only if

$$
\lambda_{i}+\lambda_{n-i+1} \geq 0
$$

$i=1,2, \ldots, n$. Additional conditions, when the graph is precisely $P$, are also discussed, but the results are incomplete.

In [75, 49, the observation of 35] is dramatically generalized. Recall that a path is a tree and that all trees are bipartite (but many non-trees are bipartite as well). All bipartite graphs are considered in [75. The main result is that $\lambda_{1} \geq \cdots \geq \lambda_{n}$ are the eigenvalues of an $n$-by- $n$ nonnegative symmetric matrix $A$ subordinate to a given bipartite graph $G$ on $n$ vertices if and only if

$$
\begin{gathered}
\lambda_{1}+\lambda_{n} \geq 0 \\
\lambda_{2}+\lambda_{n-1} \geq 0 \\
\vdots \\
\lambda_{m}+\lambda_{n-m+1} \geq 0 \\
\lambda_{m+1} \geq 0 \\
\vdots \\
\lambda_{n-m} \geq 0
\end{gathered}
$$


in which $m$ is the matching number of $G$. (Note that $\left\lfloor\frac{n}{2}\right\rfloor$ is the matching number of a path on $n$ vertices, so that the result of [35] is a special case.) Further observations about the S-NIEP for matrices subordinate to a given graph $G$ are also made.

\section{Perron Similarities}

If $S$ is an invertible matrix and there is a real, diagonal, nonscalar matrix $D$ with $S D S^{-1} \geq 0$, then $S$ is called a Perron similarity. Perron similarities were introduced by Johnson and Paparella to study the diagonalizable RNIEP and the S-NIEP.

Perron similarities were characterized in several ways in [55] and it was shown that $\mathcal{C}(S):=\left\{x \in \mathbb{R}^{n}: S D_{x} S^{-1} \geq 0\right\}$ is a polyhedral cone, i.e., a convex cone in $\mathbb{R}^{n}$ with finitely-many extremals. This was called the (Perron) spectracone of $S$, and a certain cross-section, a polytope called the (Perron) spectratope of $S$, was also discussed.

These polyhedral sets were used to verify the known necessary and sufficient conditions for the R-NIEP and the S-NIEP for orders up to four. For orders 1,2 , and 4 , it is shown that a finite number of Perron similarities are required to cover the realizable region, whereas when $n=3$, it is shown, via the relative gain array (see, e.g., [45]), that an uncountable number of Perron similarities is required to cover the realizable region.

For every $n \geq 1$, the spectracone and spectratope of $H_{n}$ were characterized, where $H_{n}$ denotes the canonical Hadamard matrix of order $2 n$. More specifically, the spectracone of $H_{n}$ is the conical hull of its rows and the spectratope of $H_{n}$ is the convex hull of its rows. The spectratope of a general, normalized Hadamard matrix was used to give a constructive proof, for Hadamard orders, of a result by Fiedler [33, that every Suleimanova spectrum is the spectrum of a symmetric nonnegative matrix (in fact, this result was strengthened to show that the constructed realizing matrix is also doubly stochastic). It is still an open problem to find a constructive proof that every Sulermanova spectrum is SNIEP-realizable. A constructive proof of the Boyle-Handelman theorem for Suleřmanova spectrum: augmenting any such spectrum with zeros up to a Hadamard order yields a spectrum realizable by a nonnegative matrix that is symmetric and doubly stochastic.

\section{Jordan structure and the NIEP's}

When there are repeated eigenvalues in a proposed spectrum, a natural question is whether Jordan structure for the repeated eigenvalues can play a role in realizability. The J-NIEP asks which particular Jordan canonical forms occur for $n$-by- $n$ nonnegative matrices and the D-NIEP is the special case of which spectra occur among diagonalizable $n$-by- $n$ nonnegative matrices. Of course, the D-NIEP and the NIEP are the same for spectra with distinct eigenvalues. It is also a simple exercise that any spectrum that is D-NIEP 
realizable by a positive matrix is also J-NIEP realizable for any Jordan canonical form possible for its eigenvalues. However, whether D-NIEP realizability always implies J-NIEP realizability for any possible Jordan form is unclear. This is so for Sulermanova spectra [15, 121, 24].

There are spectra that are realizable (in fact, R-NIEP realizable), but are not diagonalizably realizable. The smallest dimension in which this occurs is 5 . For $n \leq 4$, any realizable spectrum with (non-real) complex eigenvalues is diagonalizably realizable (the only difficulty could come from a multiple Perron root and that is easily handled by a reducibility argument). For $n \leq 4$, R-NIEP and S-NIEP realizability are the same, which settles the matter. However, for $n=5$, the spectrum

$$
3+t, 3-t,-2,-2,-2
$$

is realizable for $t>(16 \sqrt{6})^{1 / 2}-39 \approx 0.437 \ldots$, [70. However, it is diagonalizably realizable iff $t \geq 1$ (in which case it is also symmetrically realizable). Thus, for $0.437 \ldots<t<1$, this 5 -spectrum is realizable, but not diagonalizable so. This suggests that this phenomenon is fairly common.

\section{References}

[1] R. B. Bapat, T. E. S. Raghavan, Nonnegative Matrices and Applications, Cambridge University Press, Cambridge 1997.

[2] L. Benvenuti, A geometrical representation of the spectra of four dimensional nonnegative matrices, Linear Algebra Appl. 445 (2014) 162-180.

[3] L. Benvenuti. A note on eigenvalues location for trace zero doubly stochastic matrices. Electron. J. Linear Algebra 30 (2015), 599-604.

[4] A. Berman, R. J. Plemmons, Nonnegative Matrices in the Mathematical Sciencies, SIAM Publications, Philadelphia 1994.

[5] G. Bharali, O. Holtz, Functions preserving nonnegativity of matrices, SIAM J. Matrix Anal. Appl. 30 (2008) 84-101.

[6] A. Borobia, On the Nonnegative Eigenvalue Problem, Linear Algebra Appl. 223-224 (1995) 131-140.

[7] A. Borobia, Inverse Eigenvalue Problems, Handbook of Linear Algebra, chapter 20. 20-1-20-14, Chapman \& Hall/CRC, USA 2007.

[8] A. Borobia, J. Moro, On non-negative matrices similar to positive matrices, Linear Algebra Appl. 266 (1997) 365-379.

[9] A. Borobia, J. Moro, On the boundary of the set of real spectra of non-negative matrices, Linear Algebra Appl. 278 (1998) 287-293.

[10] A. Borobia, J. Moro, R. L. Soto, Negativity compensation in the nonnegative inverse eigenvalue problem, Linear Algebra Appl. 393 (2004) 73-89.

[11] A. Borobia, J. Moro, R. L. Soto, A unified view on compensation criteria in the real nonnegative inverse eigenvalue problem, Linear Algebra Appl. 428 (2008) 2574-2584.

[12] M. Boyle, D. Handelman, The Spectra of Nonnegative Matrices via Symbolic Dynamics, Ann. of Math. (2) 133(2) (1991) 249-316. 
[13] A. Brauer, Limits for the characteristic roots of a matrix, IV: Applications to stochastic matrices, Duke Math. J. 19 (1952) 75-91.

[14] R. A. Brualdi, Spectra of digraphs, Linear Algebra Appl. 432 (2010) 2181-2213.

[15] J. Ccapa, R. L. Soto, Nonnegative matrices with prescribed elementary divisors, Electron. J. Linear Algebra 17 (2008) 287-303.

[16] J. Ccapa, R. L. Soto, On spectra perturbation and elementary divisors of positive matrices, Electron. J. Linear Algebra 18 (2009) 462-481.

[17] J. Ccapa, R. L. Soto, On elementary divisors perturbation of nonnegative matrices, Linear Algebra Appl 432 (2010) 546-555.

[18] M. T. Chu, K. R. Driessel, Constructing symmetric nonnegative matrices with prescribed eigenvalues by differential equations. SIAM Journal on Mathematical Analysis 22 (1991) 1372-1387.

[19] M. T. Chu, G. H. Golub, Inverse eigenvalue problems: theory, algorithms and applications, Oxford University Press, New York 2005.

[20] P. G. Ciarlet, Some results in the theory of nonnegative matrices, Linear Algebra Appl. 1 (1968) 139-152.

[21] A. G. Cronin, Characterizing the spectra of nonnegative matrices, $\mathrm{PhD}$ thesis, School of Mathematical Sciences, University College Dublin, 2012.

[22] A. G. Cronin, T. J. Laffey, An inequality for the spectra of nonnegative matrices, Linear Algebra Appl 436 (2012) 3225-3238.

[23] D. M. Cvetković, M. Doob, H. Sachs, Spectra of Graphs, Johann Ambrosius Barth, Heidelberg 1995.

[24] R. C. Díaz, R. L. Soto, Nonnegative inverse elementary divisors problem in the left half plane, Linear Multilinear Algebra 64 (2016) 258-268.

[25] N. Dmitriev, E. Dynkin, On the characteristic numbers of a stochastic matrix, Dokl. Akad. Nauk SSSR 49 (1945) 159-162.

[26] N. Dmitriev, E. Dynkin, Eigenvalues of a stochastic matrix, Izv. Akad. Nauk SSSR Ser. Mat. 10 (1946) 167-184.

[27] D.Ž. Dokovič, Cyclic polygons, roots of polynomials with decreasing nonnegative coefficients, and eigenvalues of stochastic matrices, Linear Algebra Appl. 142 (1990) 173-193.

[28] P. D. Egleston, T. D. Lenker, S. K. Narayan, The nonnegative inverse eigenvalue problem, Linear Algebra Appl. 379 (2004) 475-490.

[29] R. Ellard, H. Šmigoc, Constructing new realisable lists from old in the NIEP, Linear Algebra Appl. 440 (2014), 218-232.

[30] R. Ellard, H. Šmigoc, Connecting sufficient conditions for the symmetric nonnegative inverse eigenvalue problem, Linear Algebra Appl. 498 (2016), 521-552.

[31] L. Elsner, R. Nabben, M. Neumann, Orthogonal bases which lead to symmetric nonnegative basis, Linear Algebra Appl. 271 (1998) 323-343.

[32] G. M. Engel, H. Schneider, S. Sergeev, On sets of eigenvalues of matrices with prescribed row sums and prescribed graph, Linear Algebra Appl. 455 (2014) 187-209.

[33] M. Fiedler, Eigenvalues of nonnegative symmetric matrices, Linear Algebra Appl. 9 (1974) 119-142.

[34] S. Friedland, On an inverse problem for nonnegative and eventually nonnegative matrices, Israel Journal of Mathematics 29 (1978) 43-60. 
[35] S. Friedland, A. A. Melkman, On the Eigenvalues of Nonnegative Jacobi Matrices, Linear Algebra Appl. 25 (1979) 239-253.

[36] G. Frobenius, Über matrizen aus positiven elementen, S. -B. K. Preuss. Akad. Wiss. Berlin, 1908 p. 471-476, 1909 p. 514-518.

[37] G. Frobenius, Über matrizen aus nicht negativen elementen, S. -B. K. Preuss. Akad. Wiss. Berlin, 1912 p. 456-477.

[38] R. Gómez, J. M. Salazar, Spanning tree invariants, loop systems and doubly stochastic matrices, Linear Algebra Appl. 432 (2010) 556-565.

[39] W. Guo, An inverse eigenvalue problem for nonnegative matrices, Linear Algebra Appl. 249 (1996) 67-78.

[40] W. Guo, Eigenvalues of nonnegative matrices, Linear Algebra Appl. 266 (1997) 261-270.

[41] W. Guo, W. Guo, Perturbing non-real eigenvalues of nonnegative matrices, Linear Algebra Appl. 426 (2007) 199-203.

[42] D. Hershkowits, Existence of matrices satisfying prescribed conditions, M. S. Thesis, Technion-Israel Inst. of Technology, Haifa, Israel, 1978.

[43] O. Holtz, M-matrices satisfy Newton's inequalities, Proc. Amer. Math. Soc. 133 (2005) n.3 711-717.

[44] R. A. Horn, C. R. Johnson, Matrix Analysis, Cambridge University Press, Cambridge 1990. Corrected reprint if the 1985 original.

[45] R. A. Horn, C. R. Johnson, Topics in Matrix Analysis, Cambridge University Press, Cambridge 1991.

[46] Suk-Geun Hwang, Sung-Soo Pyo, The inverse eigenvalue problem for symmetric doubly stochastic matrices, Linear Algebra Appl. 379 (2004) 77-83.

[47] H. Ito, A new statement about the theorem determining the region of eigenvalues of stochastic matrices, Linear Algebra Appl., 267 (1997) 241-246.

[48] C. R. Johnson, An inclusion for the field of values of a doubly stochastic matrix based on its graph, Aequationes Mathematicae 17 (1978) 305-310.

[49] C. R. Johnson, Row stochastic matrices similar to doubly stochastic matrices, Linear Multilinear Algebra 10 (1981) 113-130.

[50] C. R. Johnson, T. J. Laffey, R. Loewy, The real and the symmetric nonnegative inverse eigenvalue problems are different, Proc. AMS 124 (1996) 3647-3651.

[51] C. R. Johnson, R. B. Kellogg, A. B. Stephens, Complex eigenvalues of a nonnegative matrix with a specified graph. II, Linear Multilinear Algebra (1979), no. $2,129-143$.

[52] C. R. Johnson, C. Marijuán, M. Pisonero, Matrices and spectra satisfying the Newton inequalities, Linear Algebra Appl. 430 (2009) 3030-3046.

[53] C. R. Johnson, C. Marijuán, M. Pisonero, Symmetric Nonnegative Realizability Via Partitioned Majorization, Linear Multilinear Algebra 65 (2017), 1417-1426.

[54] C. R. Johnson, C. Marijuán, M. Pisonero, Ruling out certain 5-spectra for the symmetric nonnegative inverse eigenvalue problem, Linear Algebra Appl. 512 (2017) 129-135.

[55] C. R. Johnson, P. Paparella, Perron spectratopes and the real nonnegative inverse eigenvalue problem, Linear Algebra Appl. 493 (2016) 281-300.

[56] C. R. Johnson, P. Paparella, A matricial view of the Karpelevič Theorem, Linear Algebra Appl. 520 (2017) 1-15. 
[57] A. I. Julio, R. L. Soto, Persymmetric and bisymmetric nonnegative inverse eigenvalue problem, Linear Algebra Appl. 469 (2015) 130-152.

[58] F. Karpelevich, On the eigenvalues of a matrix with nonnegative elements, Izv. Akad. Nauk SSR Ser. Mat. 15 (1951) 361-383 (in Russian).

[59] R. Kellogg, Matrices similar to a positive or essentially positive matrix, Linear Algebra Appl. 4 (1971) 191-204.

[60] R. B. Kellogg, A. B. Stephens, Complex Eigenvalues of a Non-Negative Matrix with a Specified Graph, Linear Algebra Appl. 20 (1978) 179-187.

[61] K. H. Kim, N. S. Ormes, F. W. Roush, The spectra of nonnegative integer matrices via formal power series, J. Amer. math. Soc. 13(4) (2000) 773-806 (electronic).

[62] J. Knudsen, J. J. McDonald, A note on the convexity of the realizable set of eigenvalues for nonnegative symmetric matrices, Electron. J. Linear Algebra 8 (2001) 110-114.

[63] A. N. Kolmogorov, Markov chains with a countable number of possible states, Bull. Moskow Gosu-darstvennogo Univ. Mat. Meh., 1(3)(1937) 1-16.

[64] T. J. Laffey, Realizing matrices in the nonnegative inverse eigenvalue problem, Matrices and group representations (Coimbra, 1998), volume19 of Textos Mat. Sér. B, 21-32. Univ. Coimbra, Coimbra 1999.

[65] T. J. Laffey, Perturbing non-real eigenvalues of nonnegative real matrices, Electron. J. Linear Algebra 12 (2005) 73-76.

[66] T. J. Laffey, A constructive version of the Boyle-Handelman theorem on the spectra of nonnegative matrices, Linear Algebra Appl. 436 (2012) 1701-1709.

[67] T. J. Laffey, R. Loewy, H. Šmigoc, Nonnegative matrices that are similar to positive matrices, SIAM J. Matrix Anal. Appl. 31 (2009), no. 2, 629-649.

[68] T. J. Laffey, R. Loewy, H. Šmigoc, Power series with positive coefficients arising from the characteristic polynomials of positive matrices, Math. Ann. 364 (2016), no. 1-2, 687-707.

[69] T. J. Laffey, E. Meehan, A refinament of an inequality of Johnson, Loewy and London on nonnegative matrices and some applications, Electron. J. Linear Algebra 3 (1998) 119-128.

[70] T. J. Laffey, E. Meehan, A characterization of trace zero nonnegative $5 \times 5$ matrices, Linear Algebra Appl. 302/303 (1999) 295-302.

[71] T. J. Laffey, H. Šmigoc, Nonnegative realization of spectra having negative real parts, Linear Algebra Appl. 416 (2006) 148-159.

[72] T. J. Laffey, H. Šmigoc, Construction of nonnegative symmetric matrices with given spectrum, Linear Algebra Appl. 421 (2007) 97-109.

[73] T. J. Laffey, H. Šmigoc, On a classic example in the nonnegative inverse eigenvalue problem, Electron. J. Linear Algebra. 17 (2008) 333-342.

[74] T. J. Laffey, H. Šmigoc, Nonnegatively realizable spectra with two positive eigenvalues. Linear Multilinear Algebra 58 (2010), no. 7-8, 1053-1069.

[75] A. Leal-Duarte, C. R. Johnson, Resolution of the Symmetric Nonnegative Inverse Eigenvalue Problem for Matrices Subordinate to a Bipartite Graph, Positivity 8 (2004) 209-213. 
[76] J. Levick, R. Pereira, D.W. Kribs. The four-dimensional Perfect-Mirsky conjecture, Proceedings of the American Mathematical Society, 143 (2014), no. 5, 1951-1956.

[77] Y. J. Ley, W. R. Xu, Y. Lu, Y. R. Niu, On the symmetric doubly stochastic inevrese eigenvalue problem, Linear Algebra Appl. 445 (2014) 181-205.

[78] R. Loewy, D. London, A note on the inverse problem for nonnegative matrices, Linear and Multilinear Algebra 6 (1978) 83-90.

[79] R. Loewy, J. J. McDonald, The symmetric nonnegative inverse eigenvalue problem for $5 \times 5$ matrices, Linear Algebra Appl. 393 (2004) 275-298.

[80] R. Loewy, O. Spector, A necessary condition for the spectrum of nonnegative symmetric $5 \times 5$ matrices, Linear Algebra Appl. 528 (2017) 206-272.

[81] C. Maclaurin, A second letter to Martin Folkes, Esq.; concerning the roots of eqnarrays, with the demostration of other rules in algebra, Phil. Transactions 36 (1729) 59-96.

[82] M. Marcus, H. Minc, A Survey of Matrix Theory and Matrix Inequalities, Allyn and Bacon, Boston 1964.

[83] C. Marijuán, M. Pisonero, Relaciones entre condiciones suficientes en el problema espectral real inverso no negativo, Proceedings $V$ Jornadas de Matemática Discreta y Algorítmica: (2006) 335-342.

[84] C. Marijuán, M. Pisonero, Polinomios característicos de digrafos pesados y desigualdades de Newton, Proceedings VII Jornadas de Matemática Discreta y Algorítmica: (2010) 447-458.

[85] C. Marijuán, M. Pisonero, On Sufficient Conditions for the RNIEP and their Margins of Realizability, Electronic Notes in Discrete Mathematics 46 (2014) 201-208.

[86] C. Marijuán, M. Pisonero, R. L. Soto, A map of sufficient conditions for the real nonnegative inverse eigenvalue problem, Linear Algebra Appl. 426 (2007) 690-705.

[87] C. Marijuán, M. Pisonero, R. L. Soto, A map of sufficient conditions for the symmetric nonnegative inverse eigenvalue problem, Linear Algebra Appl. 530 (2017) 344-365.

[88] J. Mashreghi and R. Rivard, On a conjecture about the eigenvalues of doubly stochastic matrices, Linear and Multilinear Algebra 55(5) (2007) 491-498.

[89] J. J. McDonald, M. Neumann, The Soules approach to the inverse eigenvalue problem for nonnegative symmetric matrices of order $n \leq 5$, Contemp. Math. 259 (2000) 387-407.

[90] E. Meehan, Some Results On Matrix Spectra, PhD thesis (1998) National University of Ireland (Dublin).

[91] H. Minc, Inverse elememtary divisor problem for nonnegative matrices Proceedings of the American Mathematical Society, 83 n4(1981) 665-669.

[92] H. Minc, Inverse elememtary divisor problem for doubly stochastic matrices Linear and Multilinear Algegra, 11 (1982) 121-131.

[93] H. Minc, Non-Negative Matrices, John Wiley and Sons, New York 1988.

[94] L. Mirsky, Results and problems in the theory of doubly-stochastic matrices, Z. Wahrscheinlichkeitstheorie, 1 (1963) 319-334. 
[95] B. Mourad, An inverse problem for $n \times n$ symmetric doubly stochastic matrices, Inverse Problems 19 (2003) 821-831.

[96] B. Mourad, A note on the boundary of the set where the decreasingly ordered spectra of symmetric doubly stochastic matrices lie, Linear Algebra Appl. 416 (2006) 546-558.

[97] I. Newton, Arithmetica universalis: sive de compositione et resolutione arithmetica liber, 1707.

[98] G. N. de Oliveira, Sobre matrizes estocásticas e duplamente estocásticas, PhD thesis (1968), Coimbra.

[99] P. Paparella, Realizing Suleimanova-type spectra via permutative matrices, Electron. J. Linear Algebra 31 (2016) 306-312.

[100] H. Perfect, On positive stochastic matrices with real characteristic roots, Proceedings of the Cambridge Philosophical Society, 48 (1952) 271-276.

[101] H. Perfect, Methods of constructing certain stochastic matrices, Duke Math.J. 20 (1953) 395-404.

[102] H. Perfect, Methods of constructing certain stochastic matrices II, Duke Math.J. 22 (1955) 305-311.

[103] H. Perfect, L. Mirsky, Spectral properties of doubly stochastic matrices, Monatsh. Math. 69 (1965) 35-57.

[104] H. Perfect, L. Mirsky, The distribution of positive elements in doublystochastic matrices, Journal London Math. Soc 40 (1965) 689-698.

[105] O. Perron, Zur Theorie der Matrizen, Math. Ann., 64 (1907) 248-263.

[106] N. Radwan, An inverse eigenvalue problem for symmetric and normal matrices, Linear Algebra Appl. 248 (1996) 101-109.

[107] R. Reams, An inequality for nonnegative matrices and inverse eigenvalue problem, Linear and Multilinear Algebra 41 (1996) 367-375.

[108] R. Reams, Construction of trace zero symmetric stochastic matrices for the inverse eigenvalue problem, Electron. J. Linear Algebra 9 (2002) 270-275.

[109] O. Rojo, R. L. Soto, Existence and construction of nonnegative matrices with complex spectrum, Linear Algebra App. 368 (2003) 53-69.

[110] O. Rojo, R. L. Soto, Guo perturbations for symmetric nonnegative circulant matrices, Linear Algebra App. 431 (2009) 594-607.

[111] V. Romanovsky, Recherches sur les chaînes de Markoff (Premier Mèmoire), Acta Mathematica 66 (1936) 147-251.

[112] F. Salzmann, A note on the eigenvalues of nonnegative matrices, Linear Algebra Appl. 5 (1972) 329-338.

[113] H. Šmigoc, The inverse eigenvalue problem for nonnegative matrices, Linear Algebra Appl. 393 (2004) 365-374.

[114] H. Šmigoc, Construction of nonnegative matrices and the inverse eigenvalue problem, Linear Multilinear Algebra 53 (2005), no. 2, 85-96.

[115] R. L. Soto, Existence and construction of nonnegative matrices with prescribed spectrum, Linear Algebra Appl. 369 (2003) 169-184.

[116] R. L. Soto, Realizability by symmetric nonnegative matrices, Proyecciones Journal of Mathematics 24 (2005) 65-78.

[117] R. L. Soto, Realizability criterion for the symmetric nonnegative inverse eigenvalue problem, Linear Algebra Appl. 416 (2006) 783-794. 
[118] R. L. Soto (2012). Nonnegative Inverse Eigenvalue Problem, Linear AlgebraTheorems and Applications, Prof. Hassan Yasser (Ed.), InTech, DOI: 10.5772/48279. Available from: http://www.intechopen.com/books/linearalgebra-theorems-and-applications/nonnegative-inverse-eigenvalue-problem

[119] R. L. Soto, A family of realizability criteria for the real and symmetric nonnegative inverse eigenvalue problem, Numer. Linear Algebra Appl. 20 (2013) 336-348.

[120] R. L. Soto, A. Borobia, J. Moro, On the comparison of some realizability criteria for the real nonnegative inverse eigenvalue problem, Linear Algebra Appl. 396 (2005) 223-241.

[121] R. L. Soto, R. C. Díaz, H. Nina, M. Salas, Nonnegative matrices with prescribed spectrum and elementary divisors, Linear Algebra Appl. 439 (2013) 3591-3604.

[122] R. L. Soto, O. Rojo, Applications of a Brauer theorem in the nonnegative inverse eigenvalue problem, Linear Algebra Appl. 416 (2006) 844-856.

[123] R. L. Soto, O. Rojo, C. B. Manzaneda, On Nonnegative Realization of Partitioned Spectra, Electron. J. Linear Algebra 22 (2011) 557-572.

[124] R. L. Soto, O. Rojo, J. Moro, A. Borobia, Symmetric nonnegative realization of spectra, Electron. J. Linear Algebra 16 (2007) 1-18.

[125] R. L. Soto, M. Salas, C. B. Manzaneda, On Nonnegative realization of complex spectra, Electron. J. Linear Algebra 20 (2010) 595-609.

[126] G. Soules, Constructing symmetric nonnegative matrices, Linear Multilinear Algebra 13 (1983) 241-251.

[127] O. Spector, A characterization of trace zero symmetric nonnegative $5 x 5$ matrices, Linear Algebra Appl. 434 (2011), n 4, 1000-1017.

[128] H. R. Sulermmanova, Stochastic matrices with real characteristic values, Dokl. Akad. Nauk. S.S.S.R. 66 (1949) 343-345 (in Russian).

[129] J. Swift, The Location of Characteristic Roots of Stochastic Matrices, M.Sc. thesis, McGill University, Montreal, 1972.

[130] J. Torre-Mayo, M. R. Abril-Raymundo, E. Alarcia-Estévez, C. Marijuán, M. Pisonero, The nonnegative inverse eigenvalue problem from the coefficients of the characteristic polynomial. EBL digraphs. Linear Algebra Appl., 426 (2007) 729-773.

Charles R. Johnson

Department of Mathematics

College of William and Mary

Williamsburg, VA 23187-8795

USA

e-mail: crjohn@wm.edu

Carlos Marijuán

Dpto. Matemática Aplicada

E.I. Informática

Paseo de Belén 15

47011-Valladolid

Spain

e-mail: marijuan@mat.uva.es 
Pietro Paparella

Div. of Engineering \& Mathematics

University of Washington Bothell

Bothell, WA 98011-8246

USA

e-mail: pietrop@uw.edu

Miriam Pisonero

Dpto. Matemática Aplicada

E.T.S. de Arquitectura

Avenida de Salamanca 18

47014-Valladolid

Spain

e-mail: mpisoner@maf .uva.es 